CPHT-RR-046-0805

SPHT-T05/50

\title{
Perturbation theory and stability analysis for string-corrected black holes in arbitrary dimensions
}

\author{
Filipe Moura \\ Centre de Physique Théorique, École Polytéchnique \\ F91128 Palaiseau Cedex, France \\ and \\ Service de Physique Théorique, Orme des Merisiers, CEA/Saclay \\ F91191 Gif-sur-Yvette Cedex, France ${ }^{1}$ \\ fmoura@spht.saclay.cea.fr
}

\begin{abstract}
We develop the perturbation theory for $\mathcal{R}^{2}$ string-corrected black hole solutions in $d$ dimensions. After having obtained the master equation and the $\alpha^{\prime}$-corrected potential under tensorial perturbations of the metric, we study the stability of the Callan, Myers and Perry solution under these perturbations.
\end{abstract}

\footnotetext{
${ }^{1}$ Present address: Instituto Superior Técnico, Departamento de Matemática, Av. Rovisco Pais, 1049-001 Lisboa, Portugal.
} 
String theory low energy effective actions have three different types of contributions, with different origins. The classical terms come from the expansion in $\alpha^{\prime}$ (world-sheet loops). The quantum terms depend on the string coupling constant $g_{s}=\mathrm{e}^{\phi}$; they can be perturbative (coming from space-time loops) and non-perturbative. In this work we consider only the classical $\alpha^{\prime}$ corrections, neglecting any kind of string quantum correction. Both the bosonic and the heterotic string theories have corrections already at the first order in $\alpha^{\prime}$, which are at most quadratic in the Riemann tensor. In these corrections we neglect the Ricci terms, which would only contribute in a higher order in $\alpha^{\prime}$; we are only considering an effective action which is perturbative in $\alpha^{\prime}$. All these theories also have antisymmetric tensors in their massless spectra, which can always be consistently set to zero. That will be the case in the $\alpha^{\prime}$-corrected black hole solution we use in this work. Although these theories lie respectively in 26 or 10 space-time dimensions, we will consider in this article black hole space-times in generic $d$-dimensions. This way we take, as our effective action in the Einstein frame [1],

$$
\frac{1}{2 \kappa^{2}} \int \sqrt{-g}\left[R-\frac{4}{d-2}\left(\partial^{\mu} \phi\right) \partial_{\mu} \phi+\mathrm{e}^{\frac{4}{2-d} \phi} \frac{\lambda}{2} R^{\mu \nu \rho \sigma} R_{\mu \nu \rho \sigma}\right] \mathrm{d}^{d} x+\text { fermion terms },
$$

with $\lambda=\frac{\alpha^{\prime}}{2}, \frac{\alpha^{\prime}}{4}, 0$ for bosonic, heterotic and superstrings, respectively.

The corrected bosonic equations of motion for the dilaton and the graviton are, to this order,

$$
\begin{aligned}
\nabla^{2} \phi-\frac{\lambda}{4} \mathrm{e}^{\frac{4}{2-d} \phi}\left(R_{\rho \sigma \lambda \tau} R^{\rho \sigma \lambda \tau}\right) & =0, \\
R_{\mu \nu}+\lambda \mathrm{e}^{\frac{4}{2-d} \phi}\left(R_{\mu \rho \sigma \tau} R_{\nu}^{\rho \sigma \tau}-\frac{1}{2(d-2)} g_{\mu \nu} R_{\rho \sigma \lambda \tau} R^{\rho \sigma \lambda \tau}\right) & =0 .
\end{aligned}
$$

We are interested in studying the behaviour of a string-corrected black hole solution under perturbations. We will be studying these perturbations in generic $d$ spacetime dimensions [2], taking as background metric

$$
d s^{2}=-f(r) d t^{2}+f^{-1}(r) d r^{2}+r^{2} d \Omega_{d-2}^{2}
$$

with $d \Omega_{d-2}^{2}=\gamma_{i j}(\theta) d \theta^{i} d \theta^{j}, \gamma_{i j}=g_{i j} / r^{2}$ being the metric of a $(d-2)$-sphere $S^{d-2}$.

One can in general consider perturbations to the metric and any other physical field of the system under consideration. General tensors of rank at most 2 on the $(d-2)$ sphere can be uniquely decomposed in their scalar, vectorial and (for $d>4$ ) tensorial components. In this work we only consider tensorial (in $S^{d-2}$ ) perturbations to the metric, given by $h_{\mu \nu}=\delta g_{\mu \nu}$ (as we will show, we can consistently set the tensorial perturbation to the dilaton to 0 ). These perturbations are worked out in [3], where it is shown that they can be written as

$$
h_{i j}=2 r^{2}\left(y^{a}\right) H_{T}\left(y^{a}\right) \mathcal{T}_{i j}\left(\theta^{i}\right), h_{i r}=h_{i t}=0, h_{r r}=h_{t r}=h_{t t}=0
$$

with $\mathcal{T}_{i j}$ satisfying

$$
\left(\gamma^{k l} D_{k} D_{l}+k_{T}\right) \mathcal{T}_{i j}=0, D^{i} \mathcal{T}_{i j}=0, g^{i j} \mathcal{T}_{i j}=0 .
$$


$D_{i}$ is the $S^{d-2}$ covariant derivative; $\mathcal{T}_{i j}$ are the eigentensors of the $S^{d-2}$ laplacian; on the same reference [3], it is also shown that the eigenvalues are given by $-k_{T}=$ $2-l(l+d-3), l=2,3,4 \ldots$

We actually need the variation of the components of the Riemann tensor. Using the components of $h_{\mu \nu}$ given in (5) and the Palatini equation, one gets

$$
\begin{aligned}
\delta R_{i j k l} & =\left[(2 f-1) H_{T}+f \partial_{r} H_{T}\right]\left(g_{i l} \mathcal{T}_{j k}-g_{i k} \mathcal{T}_{j l}-g_{j l} \mathcal{T}_{i k}+g_{j k} \mathcal{T}_{i l}\right) \\
& +r^{2} H_{T}\left(D_{i} D_{l} \mathcal{T}_{j k}-D_{i} D_{k} \mathcal{T}_{j l}-D_{j} D_{l} \mathcal{T}_{i k}+D_{j} D_{k} \mathcal{T}_{i l}\right) \\
\delta R_{i t j t} & =\left[-r^{2} \partial_{t}^{2} H_{T}+\frac{1}{2} f f^{\prime} r^{2} \partial_{r} H_{T}+f f^{\prime} r H_{T}\right] \mathcal{T}_{i j} \\
\delta R_{i r j r} & =\left[-r \frac{f^{\prime}}{f} H_{T}--\frac{1}{2} r^{2} \frac{f^{\prime}}{f} \partial_{r} H_{T}-2 r \partial_{r} H_{T}-r^{2} \partial_{r}^{2} H_{T}\right] \mathcal{T}_{i j} \\
\delta R_{\text {abcd }} & =0 .
\end{aligned}
$$

Using the explicit form of the Riemann tensor and the variations (5) and (7-10), one can perturb the field equations (2) and (3). From (2), we are able to show that we can consistently set the dilaton perturbation $\delta \phi=0$. By perturbing (3) we are able to determine the equation for $H_{T}$, which is given by

$$
\begin{aligned}
& \left(1-2 \lambda \frac{f^{\prime}}{r}\right)\left(\partial_{t}^{2} H_{T}-f^{2} \partial_{r}^{2} H_{T}\right) \\
+ & {\left[(2-d) \frac{f^{2}}{r}-f f^{\prime}+\lambda\left(4(4-d) \frac{f^{2}}{r^{3}}(1-f)+4 \frac{f^{2}}{r^{2}} f^{\prime}+2 \frac{f}{r} f^{\prime 2}\right)\right] \partial_{r} H_{T} } \\
+ & {\left[k_{T} \frac{f}{r^{2}}\left(1+\frac{4 \lambda}{r^{2}}(1-f)\right)-2 \frac{f f^{\prime}}{r}+2(d-2) \frac{f}{r^{2}}-2(d-3) \frac{f^{2}}{r^{2}}\right.} \\
+ & \left.\lambda \frac{f}{r^{2}}\left(\frac{2+2 d}{r^{2}}-4(d-1) \frac{f}{r^{2}}+2(d-3) \frac{f^{2}}{r^{2}}-\frac{\left(f^{\prime \prime}\right)^{2}}{d-2} r^{2}\right)\right] H_{T}=0 .
\end{aligned}
$$

We now write the equation above in the form of a master equation

$$
\frac{\partial^{2} \Phi}{\partial r_{*}^{2}}-\frac{\partial^{2} \Phi}{\partial t^{2}}=: V_{T} \Phi .
$$

For that, first we write the perturbation equation in terms of the tortoise coordinate $r_{*}$, defined by $d r^{*} / d r=1 / f$. As carefully explained in [5], following the procedure introduced in [4] we derive our master function and potential:

$$
\begin{aligned}
\Phi & =\frac{H_{T}}{\sqrt{f}} \exp \left(\int \frac{\frac{f^{\prime}}{f}+\frac{d-2}{r}+\frac{4}{r^{3}}(d-4) \lambda(1-f)-\frac{4}{r^{2}} \lambda f^{\prime}-\frac{2}{r f} \lambda f^{\prime 2}}{2-\frac{4}{r} \lambda f^{\prime}} d r\right) \\
V_{T}(f) & =\left(\frac{1}{1-2 \lambda \frac{f^{\prime}}{r}}\right)^{2}\left(1+\frac{4 \lambda}{r^{2}}(1-f)\right)\left[\frac{d-4}{4 r^{2}}\left(1+\frac{4 \lambda}{r^{2}}(1-f)\right)+\frac{2 \lambda f^{\prime \prime}-1}{2 r^{2}}\right] \\
& +\frac{1}{1-2 \lambda \frac{f^{\prime}}{r}}\left[\left(k_{T}+2\right) \frac{f}{r^{2}}+2(d-3) \frac{f(1-f)}{r^{2}}+\frac{d-8}{2} \frac{f f^{\prime}}{r}-\frac{\lambda}{d-2} f\left(f^{\prime \prime}\right)^{2}\right. \\
& \left.+4 \lambda\left(k_{T}+2\right) \frac{f(1-f)}{r^{4}}+2(d-3) \lambda \frac{f(1-f)^{2}}{r^{4}}+2(d-4) \lambda \frac{f(1-f) f^{\prime}}{r^{3}}\right] \\
& +\frac{f f^{\prime}}{r}+(d-4) \frac{f^{2}}{r^{2}} .
\end{aligned}
$$


This is the potential for tensor perturbations of any kind of $R^{2}$-corrected black holes in $d$ dimensions, in terms of which the perturbation equation (11) is written as a "master equation" like (12).

To study the stability of a solution, we use the "S-deformation approach" first introduced in [3] and developed in [4]. After having obtained the potential $V_{T}(f)$, we assume that its solutions are of the form $\Phi\left(r_{*}, t\right)=e^{i \omega t} \phi\left(r_{*}\right)$, such that $\partial \Phi / \partial t=i \omega \Phi$. The master equation is then written in the Schrödinger form $A \Phi=\omega^{2} \Phi$, and a solution to the field equation is then stable if the operator $A$ is positive definite with respect to the following inner product:

$$
\langle\phi, A \phi\rangle=\int_{-\infty}^{+\infty} \bar{\phi}\left(r_{*}\right)\left[-\frac{d^{2}}{d r_{*}^{2}}+V\right] \phi\left(r_{*}\right) d r_{*}=\int_{-\infty}^{+\infty}\left[\left|\frac{d \phi}{d r_{*}}\right|^{2}+V|\phi|^{2}\right] d r_{*} .
$$

Defining $D=\frac{d}{d r_{*}}-\frac{f H_{T}}{\Phi} \frac{d}{d r}\left(\frac{\Phi}{H_{T}}\right)$ and after some algebraic tricks [5], we are left with

$$
\langle\phi, A \phi\rangle=\int_{-\infty}^{+\infty}|D \phi|^{2} d r_{*}+\int_{-\infty}^{+\infty} \frac{Q}{f}|\phi|^{2} d r_{*},
$$

with

$$
\begin{aligned}
\frac{Q}{f} & =\frac{1}{1-2 \lambda \frac{f^{\prime}}{r}} \frac{1}{r^{2}}\left[\left(k_{T}+2\right)\left(1+\frac{4 \lambda}{r^{2}}(1-f)\right)+(2 d-6)(1-f)\left(1+\frac{\lambda}{r^{2}}(1-f)\right)\right. \\
& \left.-2 r f^{\prime}-\frac{\lambda}{d-2}\left(f^{\prime \prime}\right)^{2} r^{2}\right]
\end{aligned}
$$

All that is necessary to guarantee the stability is to check the positivity of $\frac{Q}{f}$.

We considered the $R^{2}$-corrected black hole solution of the type of (4) studied in [1]. Its only free parameter is $\mu$, which is related to the classical ADM black hole mass through $m_{c l}=\frac{(d-2) A_{d-2}}{\kappa^{2}} \mu, A_{n}$ being the area of the unit $n$-sphere.

For the classical Schwarzschild-Myers-Perry solution, we have $f(r)=1-\frac{2 \mu}{r^{d-3}}$. In order to introduce the $\alpha^{\prime}$-corrections to this solution, we choose a coordinate system in which the position of the horizon, given by $r=(2 \mu)^{\frac{1}{d-3}}=: r_{H}$, is not changed. According to [1] $f(r)$ is given, in this coordinate system, by

$$
f(r)=\left(1-\left(\frac{r_{H}}{r}\right)^{d-3}\right)\left[1-\lambda \frac{(d-3)(d-4)}{2} \frac{r_{H}^{d-5}}{r^{d-1}} \frac{r^{d-1}-r_{H}^{d-1}}{r^{d-3}-r_{H}^{d-3}}\right] .
$$

This solution has as free parameters the inverse string slope $\lambda$, the black hole mass parameter $\mu$ (or, equivalently, the horizon radius $r_{H}$ ) and the spacetime dimension $d$. Since $\lambda$ is a perturbative parameter, we should take it small (say $\lambda \ll 1$ ), for the potential to make sense. For small values of $\lambda$, for each value of $d$ between 5 and 10 , and for a wide range of values of $\mu$, we have studied numerically and made plots of $\frac{Q}{f}$ as it is given by (14), and we always found positive values. From this numerical study we conclude that this solution is stable under tensor perturbations for every relevant spacetime dimension, for every value of the black hole mass.

Given the potential for the metric tensorial perturbations, we have also obtained an analytical proof of the stability and computed the absorption cross section of the black hole given by (15). For more details see [5]. 
Acknowledgements This work has been supported by a Chateaubriand scholarship from EGIDE and by fellowship BPD/14064/2003 from Fundação para a Ciência e a Tecnologia, and is part of a joint project with Ricardo Schiappa [5].

\section{References}

[1] C. G. Callan, R. C. Myers and M. J. Perry, Black Holes in String Theory, Nucl. Phys. B311 (1989) 673.

[2] H. Kodama, A. Ishibashi and O. Seto, Brane World Cosmology: Gauge Invariant Formalism For Perturbation, Phys. Rev. D62 (2000) 064022, [hep-th/0004160].

[3] A. Ishibashi and H. Kodama, Stability of Higher Dimensional Schwarzschild Black Holes, Prog. Theor. Phys. 110 (2003) 901, [hep-th/0305185]

[4] G. Dotti and R. J. Gleiser, Linear stability of Einstein-Gauss-Bonnet static spacetimes. part. I: Tensor perturbations, Phys. Rev. D 72 (2005) 044018, [arXiv:gr-qc/0503117].

[5] F. Moura and R. Schiappa, Higher-derivative corrected black holes: Perturbative stability and absorption cross-section in heterotic string theory, [arXiv:hep-th/0605001]. 